\title{
Authoring Sensory Effects in NCL
}

\author{
Fábio Barreto \\ UNILASALLE/RJ \\ Laboratório Midiacom - UFF \\ fabio.barreto at lasalle.org.br \\ fabio.barreto@midiacom.uff.br
}

\author{
Raphael Abreu \\ Institution/University Name \\ Laboratório Midiacom - UFF \\ raphael.abreu@midiacom.uff.br
Débora C. Muchaluat-Saade
Laboratório Midiacom - UFF
debora@midiacom.uff.br

\author{
Joel dos Santos \\ GPMM / CEFET/RJ \\ jsantos@eic.cefet-rj.br
}

\begin{abstract}
This work proposes an extension of NCL to provide multiple sensory effects, allowing its use for the creation of new immersive physical environments. Our contribution is to propose a new element that represents sensory effects at a high level of abstraction in a way that facilitates authoring in NCL.
\end{abstract}

\section{KEYWORDS}

Ginga, NCL, sensory effect, QoE

\section{Background}

Os efeitos sensoriais são percebidos pelos usuários através de modificações nas características da instalação física em que são executadas as aplicações mulsemídia. Desta maneira estimulando diferentes sentidos do usuário, tais como tato, olfato e até mesmo paladar. Atualmente, para realizar a integração de efeitos sensoriais em NCL, é necessário utilizar objetos com código imperativo em Lua. Para tal, o autor que for especificar tal aplicação deve compreender de programação em Lua, além de NCLua para integrar os scripts Lua ao documento NCL. Outra característica é que esses efeitos não têm conteúdo para serem transmitidos e mostrados pelo player, assim, distanciando-se da definição de objetos de mídia em NCL. Portanto é necessário oferecer uma maneira de expressar efeitos sensoriais, além de abstrair os detalhes de execução desses efeitos para o autor de aplicações NCL.

Neste contexto, esta contribuição propõe um novo módulo para a linguagem NCL, permitindo a definição do novo elemento $<$ effect $>$. Os desenvolvedores da NCL podem expressar os efeitos sensoriais de uma maneira mais abstrata, ou seja, sem entrar nos detalhes da execução. Esta abordagem facilita a autoria, incentivando assim a construção de aplicações com maior qualidade da experiência dada a maior imersão.

In: Future of Interactive Television Workshop (V WTVDI), Rio de Janeiro, Brasil. Anais Estendidos do Simpósio Brasileiro de Sistemas Multimídia e Web (WebMedia). Porto Alegre: Sociedade Brasileira de Computação, 2019.

(C)2019 SBC - Sociedade Brasileira de Computação.

ISSN: 2596-1683

\section{Proposal}

Esta contribuição propõe representar os efeitos sensoriais através de um novo elemento chamado <effect $>$ e seus atributos id, type, scent e descriptor. Tais atributos vão identificar, especificar e descrever as características da ocorrência do efeito. A propriedade type será descrita seguindo a nomenclatura de tipos de efeitos sensoriais definidos no padrão MPEG-V.

Um elemento de efeito sensorial é especializado pelo atributo type que pode ser enumerado pelos tipos padronizados MPEG-V por exemplo LightType, ScentType, TemperatureType, WindType, VibrationType, etc., ou pode identificar outros tipos de efeitos disponíveis em uma implementação do middleware Ginga-NCL. No exemplo apresentado a seguir, o atributo é usado para definir um efeito de cheiro. $\mathrm{O}$ atributo scent representa o tipo de cheiro que será disparado. Os tipos de cheiro também seguem o padrão MPEG-V.

$<$ effect id="seaScent" type="ScentType" scent = "out_at_sea" descriptor="scentDesc" />

$\mathrm{O}$ atributo descriptor referencia o elemento <descriptor $>$ que descreve características de renderização do efeito, tais como sua posição e intensidade. No exemplo a seguir, define-se uma duração de 4 segundos e a intensidade de $90 \%$, além de sua região espacial:

$<$ descriptor id="scentDesc" region="marReg" duration="4s" > $<$ descriptorParam name="intensity" value $=" 0.9$ " / >

$</$ descriptor $>$

A posição do efeito é definida através de novos atributos que foram adicionados ao elemento <region> de NCL. Este elemento pode ser usado tanto para especificar a região de exibição de mídia tradicionais ou de renderização de efeitos sensoriais. Para a localização de efeitos sensoriais, é proposto o uso do sistema de coordenadas esféricas, onde a região começa no ponto indicado pelos atributos azimuthal e polar e os atributos width e height indicam o tamanho da área a ser utilizada para renderização do efeito. Com esse sistema de coordenadas, o autor de uma aplicação não precisa se preocupar com cantos da instalação física, por exemplo, ou seu tamanho. Considere o exemplo a seguir: 
<region id="marReg" polar="-45" azimuthal="45" width="5" height="5" />

que representa a posição P na Figura 1.

Além disso, a modelagem propõe o atributo direction, onde o autor pode definir a direção do efeito usando coordenadas (intra)cardinais e também o atributo location, que segue o modelo espacial utilizado no padrão MPEG-V. A propriedade location define a localização do efeito em um espaço 3D. O valor desta propriedade é uma concatenação de posições $x, y$ e z definidas de acordo com o padrão MPEG-V. A Figura 2 ilustra todas as posições possíveis dentro do padrão. Por exemplo, para representar o efeito que ocorrerá no meio, à esquerda e na parte da frente do ambiente, usa-se a seguinte sintaxe:

<region id="marReg" location="left:middle:front" />

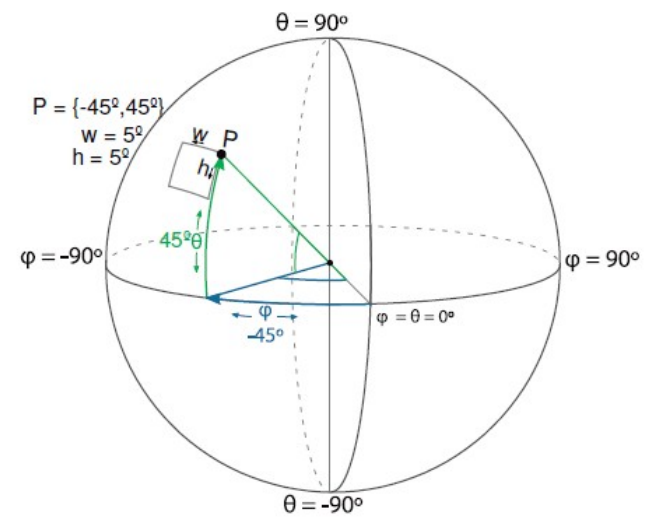

Figura 1: Posicionamento de efeitos sensoriais por coordenadas esféricas.

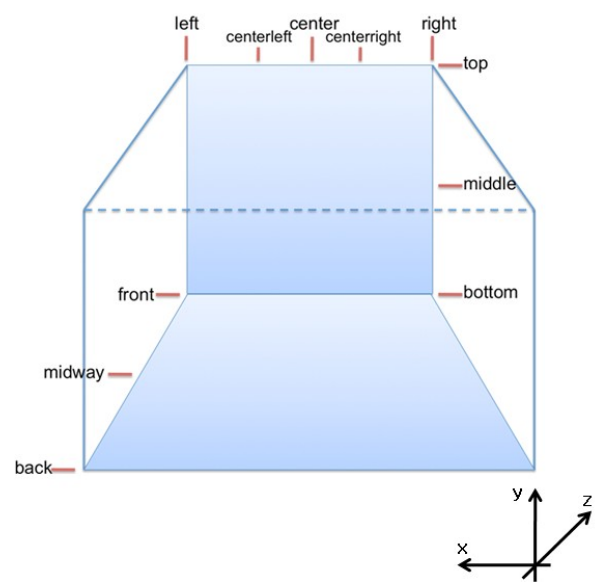

Figura 2: Posicionamento de efeitos sensoriais de acordo com o padrão MPEGV.

\section{Use Cases}

Uma exposição multissensorial interativa para um museu pode ser desenvolvida em NCL com a modificação proposta. O guia da exposição inicia um conteúdo multimídia relativo a praia. Desta forma, pode-se criar um conector para disparar o efeitos de cheiro do mar, vento, luminosidade e temperatura no momento em que aparece o mar no vídeo. A Figura 3 mostra a visão temporal desta desta aplicação enquanto a Figura 4 mostra a visão estrutural desta aplicação.

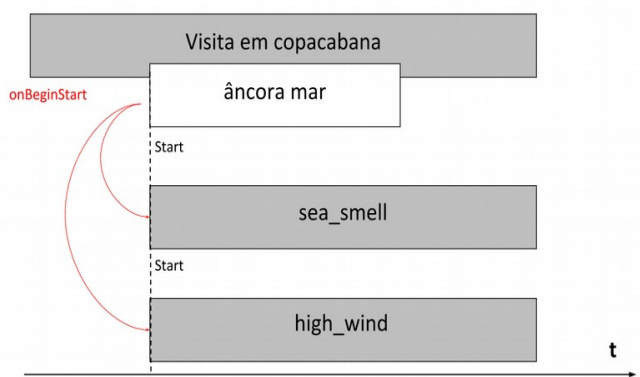

Figura 3: Visão temporal da aplicação que apresenta um vídeo da praia em um ambiente imersivo.

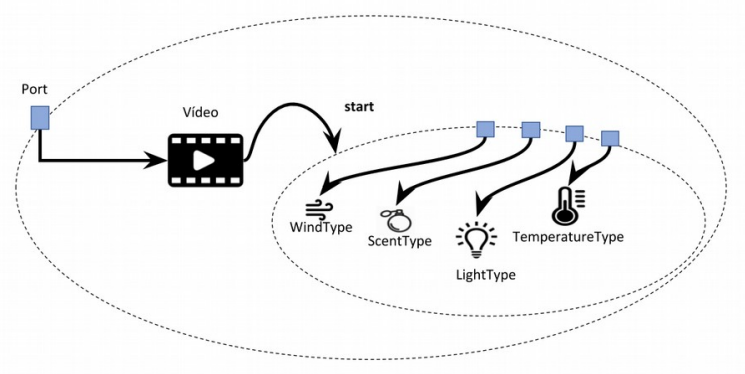

Figura 4: Visão estrutural de uma aplicação com efeitos sensoriais para aumentar a imersão do usuário em um vídeo que apresenta a praia. 